\title{
Emergent central sleep apnea during CPAP therapy-clinical implications
}

\author{
M. Jeffery Mador \\ Department of Medicine, Division of Pulmonary, Critical Care and Sleep Medicine, Western New York Veterans Affairs Healthcare System, \\ University at Buffalo, NY, USA \\ Correspondence to: M. Jeffery Mador, MD. Associate Professor of Medicine, Division of Pulmonary, Critical Care and Sleep Medicine, University at \\ Buffalo, NY, USA. Email: mador@buffalo.edu. \\ Provenance: This is an invited Editorial commissioned by Section Editor Dr. Ning Ding (Department of Respiratory and Critical Care Medicine, The \\ First Affiliated Hospital of Nanjing Medical University, Nanjing, China). \\ Comment on: Liu D, Armitstead J, Benjafield A, et al. Trajectories of Emergent Central Sleep Apnea during Continuous Positive Airway Pressure \\ Therapy. Chest 2017. [Epub ahead of print].
}

Submitted Sep 18, 2017. Accepted for publication Sep 25, 2017.

doi: $10.21037 /$ jtd.2017.09.131

View this article at: http://dx.doi.org/10.21037/jtd.2017.09.131

CPAP emergent central sleep apnea (CSA) (classified as treatment emergent CSA) is a well-recognized phenomenon that has been included in the current edition of the International Classification of Sleep Disorders (1). It is defined as the presence of primarily obstructive sleep apnea (OSA) on the diagnostic sleep study, significant resolution of obstructive events with CPAP titration but emergence or persistence of central events during PAP treatment with a central apnea index $(\mathrm{CAI}) \geq 5 / \mathrm{hr}$ and greater than $50 \%$ of events being central and the results cannot be better explained by another CSA disorder. The European Respiratory Society has recently clarified the diagnostic criteria (2). It refines the definition to state that central events should be rare on the diagnostic study which I believe should be defined as a CAI less than $5 / \mathrm{hr}$. If the CAI is greater than $5 / \mathrm{hr}$ on the diagnostic study then a CAI greater than $5 / \mathrm{hr}$ during the titration study is really CPAP resistant CSA (defined as treatment resistant CSA by the European Respiratory Society) and not true CPAP emergent CSA.

CPAP emergent CSA's prevalence is highly variable in the literature. In a recent systematic review and metaanalysis, nine studies were identified that addressed the prevalence of CPAP emergent CSA (3). Prevalence varied from $5.0 \%$ to $20.3 \%$ with an aggregate point estimate of $8.4 \%$. There were a total of 4,375 subjects studied. CPAP emergent sleep apnea was diagnosed during the CPAP titration study or during the titration portion of a split night study. The prevalence likely varies based on differences in predisposing factors between patient populations and differences in prevalence of a CAI greater than $5 / \mathrm{hr}$ during the diagnostic study as in the past these patients often have been included in estimates of CPAP emergent CSA provided the majority of events during the diagnostic study were obstructive.

CPAP emergent CSA usually resolves over time $(4,5)$. However, it can also persist over time (defined as CPAP persistent CSA) and can develop later in the course of PAP treatment despite being absent during the initial diagnostic sleep study (defined as emergent CSA) (5).

In a recent study, a different approach to examining CPAP emergent CSA was employed (6). These authors queried a large administrative commercial data base (Resmed) to better understand the prevalence of this condition. They took a sample of patients in the United States who were participating in CPAP telemonitoring via Resmed designed for monitoring compliance with CPAP therapy (autoCPAP or fixed pressure). The PAP devices employed in this study use the forced oscillation technique to determine airway patency and thus differentiate central from obstructive apneas. Patients were included if they used CPAP for at least 90 days and had at least one day of more than 1 hour of usage during week 1 and week 13. A sample of 133,066 patients meeting this criterion was obtained. They then could determine the prevalence of transient CPAP emergent CSA (CAI $\geq 5 /$ hr during week 1 but not week 13 ), 
persistent CPAP emergent CSA (CAI $\geq 5 / \mathrm{hr}$ during week 1 and week 13) and late CPAP emergent CSA (CAI less than $5 /$ hr during week 1 but $\geq 5 / \mathrm{hr}$ during week 13 ). They found that $3.5 \%$ of patients had CPAP emergent CSA, in $55.1 \%$ it was transient, $25.2 \%$ had persistent and $19.7 \%$ had late CPAP emergent CSA.

Patients with CPAP emergent CSA were older and had larger air leak during CPAP therapy. Patients who developed CPAP emergent CSA were more likely to stop CPAP therapy than those that did not.

This is an important and clever study. They utilized an existing commercial database to look at the time course of evolution of CPAP emergent CSA in a huge group of patients that was 30 times larger than all the patients in the prior studies examining CPAP emergent CSA identified in the recent meta-analysis (3). They also obtained data continuously over 90 days to provide more definitive evidence of the time course of evolution of CPAP emergent CSA than has hitherto been available.

However, like all database studies there are limitations. The database did not contain any clinical information about patient comorbidities, or results from the diagnostic sleep study or even confirmation that the patient truly had OSA so that the prevalence of known risk factors for CSA like heart failure, atrial fibrillation or opiate usage could not be determined. Likewise the presence of central events during the diagnostic study is unknown. These factors if present could increase the prevalence of CSA. However, the prevalence of CPAP emergent CSA from this study of $3.5 \%$ was actually less than half of the point prevalence of $8.4 \%$ derived from the Meta-analysis of prior studies. This could reflect the fact that the present study is a more representative sample of the sleep apnea population at large. Alternative explanations are equally plausible. Some patients may have been put on more complex positive pressure devices such as ASV when CPAP emergent sleep apnea was identified during the CPAP titration study and thus would never have received CPAP and would not have been included in this analysis. This might be a very important reason for the discrepancy between studies.

Are there any technical factors that might lead to an underestimation of CSA? The device uses the forced oscillation technique to distinguish central from obstructive apneas. The accuracy of this approach has been evaluated for detection of obstructive events (7) but not to my knowledge for the evaluation of central events. Theoretically it should be a good way to distinguish central from obstructive events but experimental validation would be helpful. In a prior study, the accuracy of a different CPAP device's estimate of AHI was evaluated by simultaneous smart card reading of events (each event sent a spike to the polysomnogram to identify a smart card event) which could then be compared event by event with polysomnographic derived events (8). No such analysis appears to have been published to validate the accuracy of central apnea detection by the forced oscillation technique. The smart card does not distinguish wakefulness from sleep. Thus the denominator for the AHI estimate may be falsely high as it represents total recording time rather than total sleep time and this could artefactually lower the CAI. Events could also be detected during wakefulness which would artefactually increase the AHI and possibly the CAI. However, detecting events during wakefulness is likely more of an issue when the overall $\mathrm{AHI}$ is low where the smart card on average can slightly overestimate the $\mathrm{AHI}$ while when the $\mathrm{AHI}$ is high on average (as it would be if CPAP emergent CSA was present) the smart card usually underestimates the AHI likely due to using total recording time as the denominator for calculating the AHI (9). Thus, there are a lot of reasons to be uncertain about the accuracy of this studies estimate of the prevalence of CPAP emergent CSA.

What about the temporal time course of CPAP emergent CSA? This study shows that $20 \%$ of patients developed CPAP emergent CSA over time. The reason why this occurs is not immediately apparent but it is a fascinating finding and deserves further investigation. Twenty five percent of patients had persistent CPAP emergent sleep apnea. However, we do not know how many of these patients had comorbidities that predispose to CSA or who had an increased CSA index during the diagnostic study so what percentage of these patients truly had persistent CPAP emergent CSA is unknown. Finally the remainder of the patients had their CPAP induced CSA resolve over time consistent with prior studies $(4,5)$.

What about risk factors for the development of CPAP emergent CSA. Prior studies have identified male sex (3 of 7 studies), age (1 of 8 studies), high baseline AHI (4 of 8 studies), high baseline CAI (4 of 7 studies), presence of heart failure or ischemic heart disease (1 of 8 studies), high baseline arousal index (1 of 6 studies), high CPAP pressure (1 of 5 studies, one study with equivocal results) and low BMI (1 of 8 studies) (3). In this study the only potential risk factors measured were age and CPAP pressure. Older age was found to be a significant risk factor for CPAP emergent CSA. CPAP was minimally but significantly higher in those with CPAP emergent CSA but the magnitude of difference was so small (less than 
$\left.1 \mathrm{cmH}_{2} \mathrm{O}\right)$ that this difference is not clinically meaningful. The authors also found that air leak was significantly higher in patients with CPAP emergent CSA as has been observed in a prior study where the authors speculated that this observation might be mechanistically important (by leading to greater sleep disruption and/or greater carbon dioxide clearance both of which could promote central events) (10). Further study to determine whether efforts to reduce air leak result in amelioration of CPAP emergent CSA (persistent or late emergent) are warranted.

Finally the authors showed that subjects who developed CPAP emergent CSA had lower CPAP compliance during the first 90 days and were more likely to stop using CPAP. These effects were smallest in those whose CPAP emergent CSA resolved over time, larger in those with persistent CPAP emergent CSA and greatest in those whose CPAP emergent CSA emerged later during CPAP treatment. These data do not confirm that CPAP emergent CSA is responsible for this reduced compliance as an unmeasured confounder could also be responsible. Nevertheless, it does raise the possibility that it might be responsible for CPAP discontinuation in some patients and therefore treatment might be helpful in patients with persistent or late emergence of CPAP emergent CSA. In this regard ASV is superior to other modalities for treatment of CPAP emergent CSA (11).

\section{Acknowledgements}

None.

\section{Footnote}

Conflicts of Interests: The author has no conflicts of interest to declare.

\section{References}

1. American Academy of Sleep Medicine. International

Cite this article as: Mador MJ. Emergent central sleep apnea during CPAP therapy-clinical implications. J Thorac Dis 2017;9(11):4182-4184. doi: 10.21037/jtd.2017.09.131
Classification of Sleep Disorders. 3rd ed. Darien, IL: American Academy of Sleep Medicine; 2014.

2. Randerath W, Verbraecken J, Andreas S, et al. Definition,d iscrimination, diagnosis and treatment of central breathing disorders during sleep. Eur Respir J 2017;49. pii: 1600959.

3. Nigam G, Pathak C, Riaz M. A systematic review on prevalence and risk factors associated with treatmentemergent central sleep apnea. Ann Thorac Med 2016;11;202-10.

4. Javaheri S, Smith J, Chung E. The prevalence and natural history of complex sleep apnea. J Clin Sleep Med 2009;5:205-11.

5. Cassel W, Canisius S, Becker HF, et al. A prospective polysomnographic study on the evolution of complex sleep apnoea. Eur Respir J 2011;38:329-37.

6. Liu D, Armitstead J, Benjafield A, et al. Trajectories of Emergent Central Sleep Apnea during Continuous Positive Airway Pressure Therapy. Chest 2017. [Epub ahead of print].

7. Badia JR, Farré R, Montserrat JM, et al. Forced oscillation technique for the evaluation of severe sleep apnoea/hypopnoea syndrome: a pilot study. Eur Respir J 1998;11:1128-34.

8. Berry RB, Kushida CA, Kryger MH, et al. Respiratory event detection by a positive airway pressure device. Sleep 2012;35:361-7.

9. Desai H, Patel A, Patel P, et al. Accuracy of autotitrating CPAP to estimate the residual Apnea-Hypopnea Index in patients with obstructive sleep apnea on treatment with autotitrating CPAP. Sleep Breath 2009;13:383-90.

10. Montesi SB, Bakker JP, Macdonald M, et al. Air leak during CPAP titration as a risk factor for central sleep apnea. J Clin Sleep Med 2013;9:1187-91.

11. Morgenthaler TI, Kuzniar TJ, Wolfe JF, et al. The complex sleep apnea resolution study: a prospective randomized controlled trial of continuous positive airway pressure versus adaptive servoventilation therapy. Sleep 2014;37:927-34. 